\title{
MicroRNA-365a-3p promotes tumor growth and metastasis in laryngeal squamous cell carcinoma
}

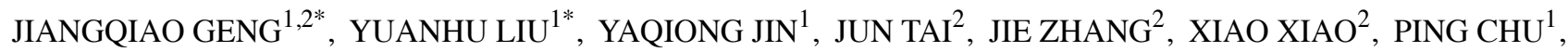 \\ YONGBO YU ${ }^{1}$, SHENG CAI WANG ${ }^{2}$, JIE LU $^{1}$, SHUJING HAN ${ }^{1}$, JIN SHI $^{1}$, YONGLI GUO ${ }^{1}$ and XIN NI ${ }^{1,2}$ \\ ${ }^{1}$ Beijing Key Laboratory for Pediatric Diseases of Otolaryngology, Head and Neck Surgery, \\ Beijing Pediatric Research Institute, Beijing Children's Hospital, Capital Medical University; ${ }^{2}$ Department of Otolaryngology, \\ Head and Neck Surgery, Beijing Children's Hospital, Capital Medical University, Beijing 100045, P.R. China
}

Received October 9, 2015; Accepted November 10, 2015

DOI: $10.3892 / o r .2016 .4617$

\begin{abstract}
MicroRNAs (miRNAs) are increasingly recognized as oncogenes or tumor suppressors in laryngeal squamous cell carcinoma (LSCC). In this study, we analyzed the roles of miR-365a-3p, miR-143-5p, and miR-494-3p in LSCC using Annexin V/propidium iodide double staining and flow cytometry, along with a Transwell migration and invasion assay. The results showed that miR-365a-3p inhibitor significantly facilitated cell apoptosis and suppressed cell cycle progression, migration, and invasion in Hep-2 cells. However, miR-143-5p and miR-494-3p had no such influences. We then investigated the role of miR-365a-3p in LSCC in vivo and found that miR-365a-3p inhibitor suppressed LSCC xenograft tumor growth and metastasis in xenograft mouse models. Moreover, miR-365a-3p inhibitor significantly decreased the expression of p-AKT (Ser473), which indicated that miR-365a-3p can mediate PI3K/AKT signaling pathway transduction via p-AKT (Ser473) in LSCC. The data suggest that miR-365a-3p may act as an oncomiR and may promote growth and metastasis in LSCC via the PI3K/AKT signaling pathway, and thus miR-365a-3p may be a potential therapeutic target for treatment of LSCC.
\end{abstract}

Correspondence to: Professor Xin Ni, Beijing Key Laboratory for Pediatric Diseases of Otolaryngology, Head and Neck Surgery, Beijing Pediatric Research Institute, 56 Nanlishi Road, Beijing 100045, P.R. China

E-mail: xinnibch@163.com

Dr Jun Tai, Department of Otolaryngology, Head and Neck Surgery, Beijing Children's Hospital, Capital Medical University, 56 Nanlishi Road, Beijing 100045, P.R. China

E-mail: trenttj@163.com

${ }^{*}$ Contributed equally

Key words: miR-365a-3p, miR-143-5p, miR-494-3p, laryngeal squamous cell carcinoma, AKT

\section{Introduction}

Laryngeal squamous cell carcinoma (LSCC) is the most common type of laryngeal carcinoma (LC) and accounts for more than $90 \%$ of LC cases. In 2015, the estimated number of new cases of LC was 13,560 in the United States, and about 3,640 Americans were likely to die from LC (1). During the past 2 decades, clinicians have seen a worrying decline in the survival rate of LC patients, partly due to lymph node metastases (LNM). Recent studies showed that the 5-year overall survival was $39-44 \%$ in patients with advanced LC, and the 2-year local relapse rate was $27.5 \%$ in stage III LC $(2,3)$. Although a variety molecules associated with the oncogenesis and the treatment of LSCC have been identified, the potential therapeutic targets and the precise molecular mechanisms of LSCC proliferation and metastasis remain to be elucidated.

In the past few years, miRNAs have been identified in most carcinomas and have become potential biomarkers for cancer diagnosis and treatment (4-6). miRNAs are small, single-stranded non-coding RNA molecules that regulate gene expression by binding to miRNA at the 3'-untranslated region (7). As of May 2014, 2,578 miRNAs have been identified in the human genome (4). Deregulated miRNAs have been shown to play pivotal roles in the multistep process of carcinogenesis via regulated oncogenes or tumor suppressor genes. For example, miR-10b and miR-320b enhance cell proliferation and invasion in pancreatic cancer and colorectal cancer, respectively $(8,9)$, and miR-218 and miR-195 inhibit cell proliferation, migration, and invasion in gliomas and non-small cell lung cancer $(10,11)$.

miRNAs also play important roles in LSCC $(12,13)$. miR-27a was significantly upregulated in LSCC, promoted cell viability and colony formation, and repressed apoptosis by targeting PLK2 in Hep-2 cells (13). Our previous study (14) reported the identification via miRNA microarray and verification by real-time PCR (RT-PCR) of $10 \mathrm{miRNAs}$ that were expressed differentially in LSCC patients with vs. those without LNM, including 9 upregulated miRNAs (miR-365a-3p, miR-143-5p, miR-634, miR-223-3p, miR-409-5p, miR-1224-3p, miR-192-5p, miR-30d-5p, and miR-1249-3p) and 1 downregulated miRNA (miR-494-3p). 
Based in part on findings from our previous study, we analyzed the effects of miR-365a-3p, miR-143-5p, and miR-494-3p in LSCC in vitro. However, only miR-365a-3p can affect cell apoptosis, mitosis, migration, and invasion. We also found that miR-365a-3p promoted LSCC xenograft tumor growth and metastases in liver and hepatic lymph nodes in mouse models. Moreover, miR-365a-3p was demonstrated to mediate the PI3K/AKT signaling pathway via p-AKT (Ser473) in LSCC.

\section{Materials and methods}

Cell culture and transient infection. Human Hep-2 cell lines (American Type Culture Collection, ATCC; Manassas, VA, USA) were cultured in RPMI-1640 medium (Gibco-Life Technologies, Carlsbad, CA, USA) with $10 \%$ fetal bovine serum at $37^{\circ} \mathrm{C}$ and $5 \% \mathrm{CO}_{2}$ in a humidified-atmosphere incubator. For overexpression and inhibition of miRNAs (all from GE Healthcare Dharmacon, Lafayette, CO, USA), miR-365a-3p inhibitor (IH-300666-05-0002), miR-143-5p inhibitor (IH-301057-02-0002), miR-494-3p mimic (C-300761-05-0002), normal control (NC)-inhibitor (IN-001005-01-05), and NC-mimic (CN-001000-01-05) were transiently transfected in Hep-2 cells by Lipofectamine 2000 (Invitrogen-Life Technologies, Carlsbad, CA, USA) following the manufacturer's protocol.

RNA extraction and real-time PCR analysis. Total RNAs of the transfected Hep-2 cells were extracted with TRIzol (Invitrogen-Life Technologies) according to the manufacturer's protocol. Single-stranded cDNA of the miRNAs was synthesized by reverse transcription using the miScript Reverse Transcription kit (Qiagen, Valencia, CA, USA). The expression level of transfected miRNAs was assessed with RT-PCR (TaqMan MicroRNA Assay; Life Technologies). The PCR primers for miR-365a-3p (sense, GCCCCTAAAAATCCTT and antisense, GTGCAGGGTCCGAGGT); miR-143-5p (sense, AAAAGAAAGAAAACACCC and antisense, GTGC AGGGTCCGAGGT); miR-494-3p (sense, GAAACATACAC GGGAAACC and antisense, GTGCAGGGTCCGAGGT); and U6 (sense, TGCGGGTGCTCGCTTCGCAGC and antisense, CCAGTGCAGGGTCCGAGGT) were obtained from Life Technologies. U6 served as an internal reference. The relative expression levels of miR-365a-3p, miR-143-5p, and miR-494-3p in Hep-2 cells were calculated using the $2^{-\Delta \Delta C t}$ method.

Cell apoptosis assay. Hep-2 cells were seeded in 6-well plates, grown to about $80 \%$ confluence, and transiently transfected with the prepared miRNA inhibitors and mimics. NC-mimic and NC-inhibitor were used as controls. The transfected cells were trypsinized, washed twice in prechilled phosphate-buffered saline (PBS), and collected after transfection at 48 and $72 \mathrm{~h}$. Subsequently, the cells were resuspended with $300 \mu \mathrm{l}$ of $1 \mathrm{X}$ binding buffer. Then, $5 \mu \mathrm{l}$ of Annexin V-FITC conjugate and $5 \mu \mathrm{l}$ of propidium iodide (PI) solution (both from BD Biosciences, San Diego, CA, USA) were added, and cells were incubated at room temperature for $15 \mathrm{~min}$ in the dark. Flow cytometric analysis for cell apoptosis was performed with a FACSCalibur flow cytometer (BD Biosciences) according to the manufacturer's instructions.
Cell cycle assay. The Hep-2 cells were treated according to the procedures for cell apoptosis assay. NC-mimic and $\mathrm{NC}$-inhibitor were used as controls. Subsequently, the transfected cells were resuspended with $400 \mu \mathrm{l}$ PBS and fixed with prechilled $80 \%$ ethanol at $4^{\circ} \mathrm{C}$ for 24 to $48 \mathrm{~h}$ for cell cycle analysis. Then, the fixed cells were washed with PBS and resuspended in staining solution (Boehringer Mannheim, Mannheim, Germany) containing $50 \mu \mathrm{g} / \mathrm{ml}$ of RNase A and $65 \mu \mathrm{g} / \mathrm{ml}$ of PI solution and incubated at $37^{\circ} \mathrm{C}$ for $30 \mathrm{~min}$. Cell cycle flow cytometric analysis was performed with a FACSCalibur flow cytometer.

Transwell migration and invasion assay. For the migration assay, the transiently transfected Hep-2 cells with the miRNA inhibitors and mimics described above were seeded in the upper Transwell chamber (Sigma-Aldrich, St. Louis, MO, USA) coated with $8-\mu \mathrm{m}$ pore Transwells (Millipore, Billerica, MA, USA). After incubation for $24 \mathrm{~h}$, the non-migrated cells in the upper chamber were removed. The invaded cells were fixed with methanal, stained with Giemsa staining solution, and then counted by an inverted light microscope. The Hep-2 cells invasion assays were performed using the Matrigel invasion chamber (Sigma-Aldrich) according to the manufacturer's protocol. The seeding, staining, and counting of Hep-2 cells were performed as the migration assay. In the above two assays, the medium of upper Transwell chamber contains serum-free culture, and of the lower chamber contains $10 \%$ fetal bovine serum served as the chemoattractant.

Lentivirus-mediated inhibition of $m i R-365 a-3 p$. To establish the stable miR-365a-3p and miR-365a-3p inhibitor cell lines, Hep-2 cells were transfected with NC-pLenti6/TR inhibitor (Lv-NC-inhibitor) and pLenti6/TR miR-365a-3p inhibitor (Lv-miR-365a-3p inhibitor), respectively, followed by selection for 42 days in fresh complete medium supplemented with $3 \mu \mathrm{g} / \mathrm{ml}$ puromycin (Invitrogen-Life Technologies). Single colonies were selected and amplified, and the expression level of miR-365a-3p was detected by RT-PCR.

Analysis of tumorigenesis and metastatic potential of $m i R-365 a-3 p$ in vivo. For the in vivo tumorigenesis assay, $2 \times 10^{6}$ stable transfected Hep-2 cells with Lv-NC-inhibitor were injected into the enterocoelia of 10 nude mice (Laboratory Animal Center of the Capital Medical University, Beijing, China), aged 6 weeks, so were the $2 \times 10^{6}$ stable transfected Hep-2 cells with Lv-miR-365a-3p inhibitor. The mice were sacrificed on the day 42 after injection, and the final tumors were isolated. Tumor size was measured with a digital caliper, and tumor volume was calculated with the formula: tumor volume $=\left(\right.$ length $\mathrm{x}$ width $\left.{ }^{2}\right) \times 0.5$. For the tumor-metastasis assay in vivo, all the organs of the sacrificed mice were examined at necropsy. Lungs, livers, and the corresponding lymph nodes were stained with hematoxylin and eosin and were examined by two pathologists.

All mice used in this experiment were bred and maintained in sterile cages and were handled according to the National Institutes of Health Animal Care and Use Committee Regulations. All experimental procedures were approved by the Animal Care and Use Committee of Capital Medical University (Beijing, China). 
A

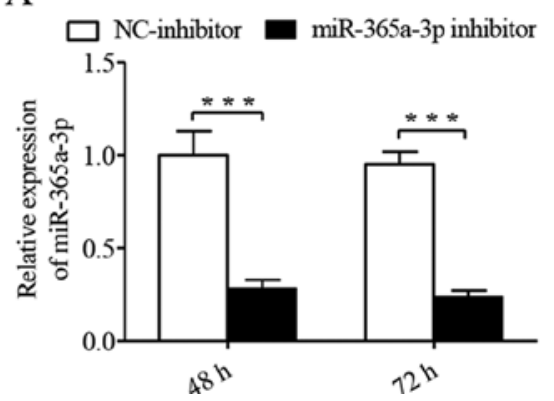

B

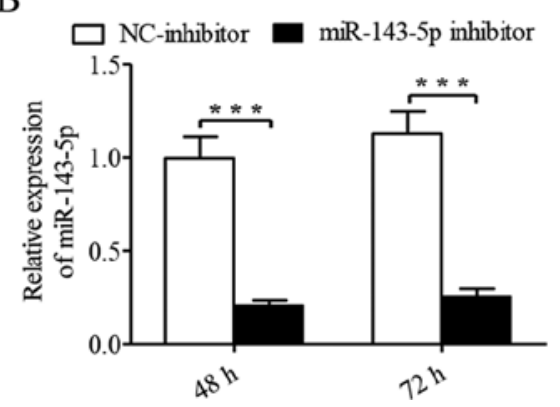

C

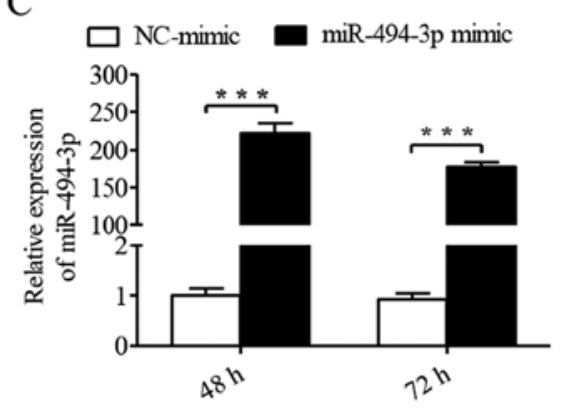

Figure 1. The expression level of miRNAs in Hep-2 cells was measured using RT-PCR. (A and B) The expression levels of miR-365a-3p and miR-143-5p in Hep-2 cells transfected with NC-inhibitor, miR-365a-3p inhibitor, and miR-143-5p inhibitor, respectively. (C) The levels of 494-3p in Hep-2 cells transfected with NC-mimic and miR-494-3p mimics. ${ }^{* * *} \mathrm{P}<0.001$ vs. the control.

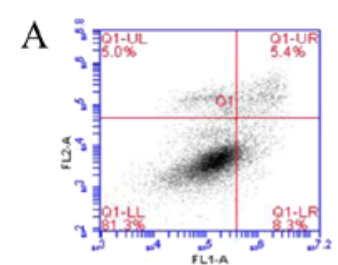

NC-inhibitor (48 h)

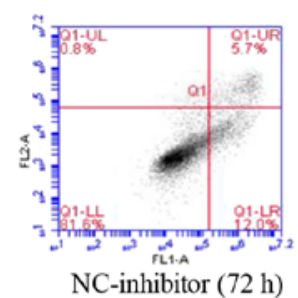

C
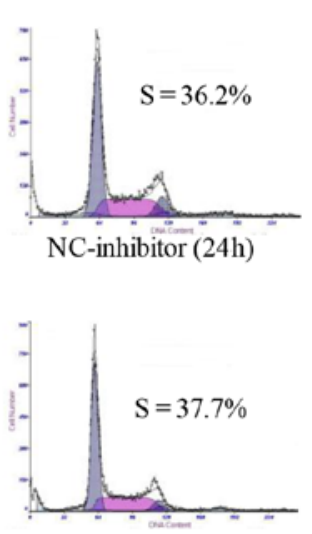

$\mathrm{NC}$-inhibitor (48 h)

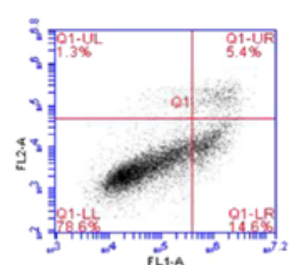

miR-365a-3p inhibitor (48 h)

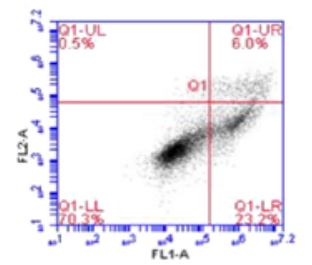

miR-365a-3p inhibitor (72 h)
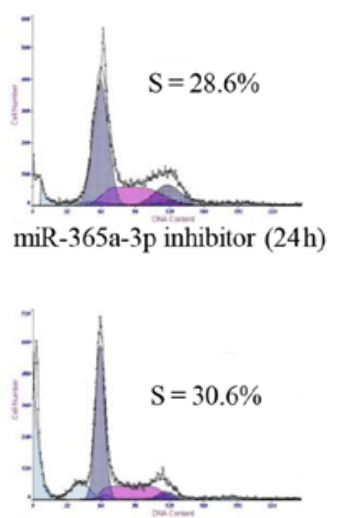

miR-365a-3p inhibitor (48 h)
B

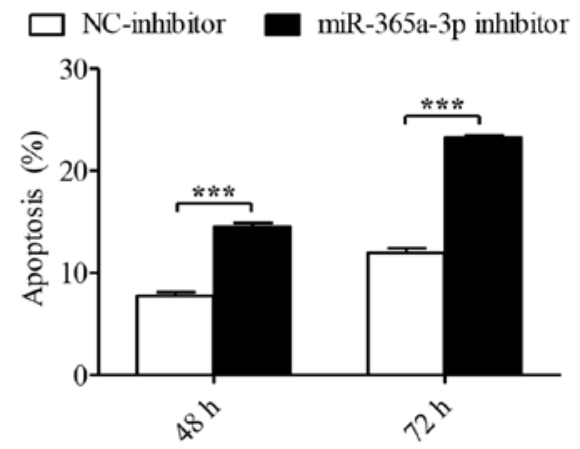

D

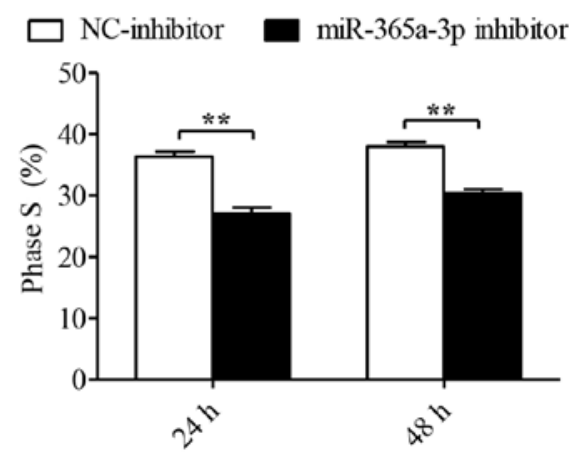

Figure 2. The effects of miR-365a-3p inhibitor on regulating LSCC cell apoptosis and mitosis measured by flow cytometry. (A) miR-365a-3p inhibitor facilitated the apoptosis of Hep-2 cells after transfection with NC-inhibitor and miR-365a-3p inhibitor. (B) The apoptosis percentages of infected Hep-2 cells at 48 and $72 \mathrm{~h}$ were counted and are shown in the histogram. ${ }^{* * *} \mathrm{P}<0.001$ vs. the control. (C) Fewer Hep-2 cells infected with miR-365a-3p inhibitor were in $\mathrm{S}$ phase at 24 and $48 \mathrm{~h}$. (D) The percentages of infected Hep- 2 cells in S1 phase at 24 and $48 \mathrm{~h}$ were counted and are shown in the histogram. ${ }^{* *} \mathrm{P}<0.01$ vs. the control.

Protein extraction and western blot analysis. Cells were lysed in RIPA buffer with a protease inhibitor cocktail (both from Millipore). Protein was extracted and analyzed by standard western blot analyses. The primary antibodies, including total ERK, p-ERK1/2, total AKT, p-Akt (Ser473), and p-Akt (Thr308) were obtained from Life Technologies. GAPDH was used as an endogenous normalizer. The membrane was incubated with alkaline phosphatase secondary antibodies
(Thermo Fisher Scientific, Waltham, MA, USA). Then the intensities of enhanced bands of immunoreactive protein was quantified (Image-Pro Plus 6.0; Media Cybernetics, Rockville, MD, USA).

Statistical analyses. All of the experiments were repeated in triplicate, and one representative experiment was selected for data analysis. The statistical analyses were performed with 
A

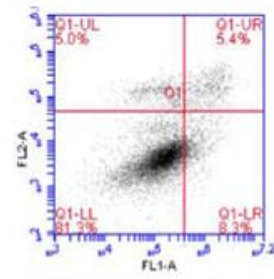

$\mathrm{NC}$-inhibitor (48 h)

B

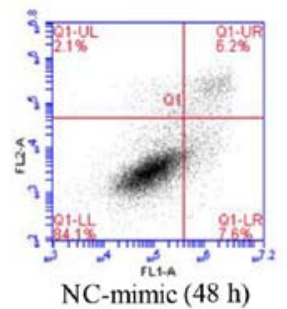

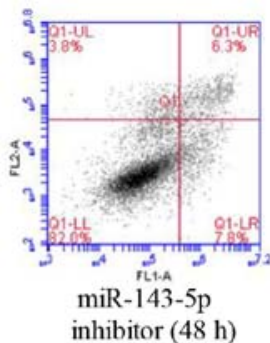

inhibitor (48 h)

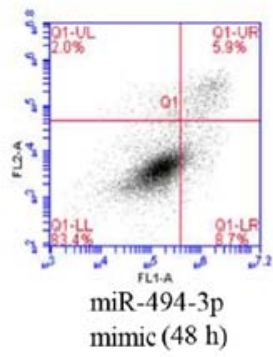

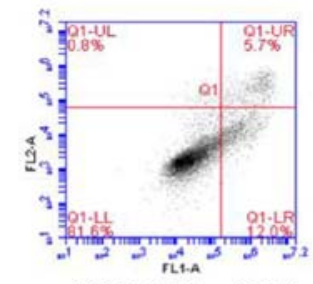

$\mathrm{NC}$-inhibitor (72 h)

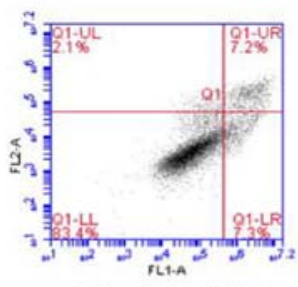

NC-mimic (72 h)

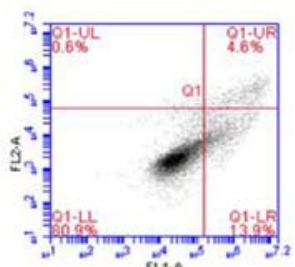

miR-143-5p

inhibitor (72 h)

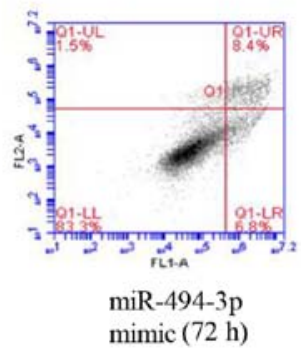

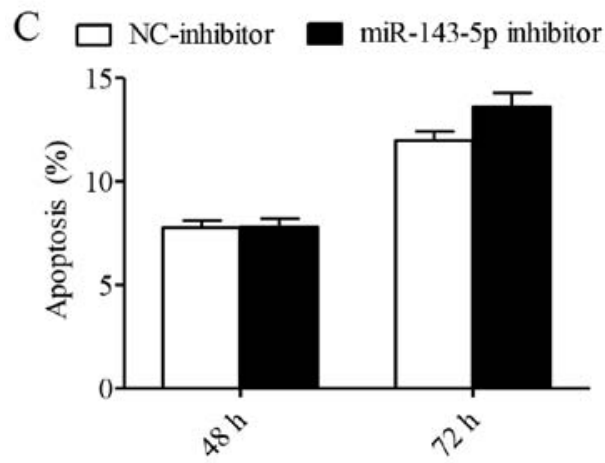

D

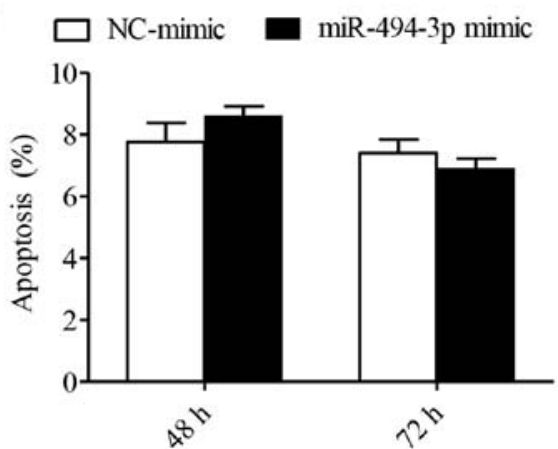

Figure 3. The apoptosis assay of infected Hep-2 cells with miR-143-5p inhibitor and miR-494-3p mimic based on flow cytometry analyses. (A and B) The apoptosis assay of infected Hep-2 cells with miR-143-5p inhibitor and miR-494-3p mimic was performed at 48 and 72 h using flow cytometry analyses. (C and D) The apoptosis ratios of homologous Hep-2 cells infected with miR-143-5p inhibitor and miR-494-3p mimic at 48 and $72 \mathrm{~h}$ were counted and are shown in the histograms.

SPSS 17.0 (IBM, Chicago, IL, USA). The statistical significance was tested using two-tailed Student's t-test. A p-value of $<0.05$ was considered statistically significant.

\section{Results}

miR-365a-3p inhibitor facilitates apoptosis and suppresses mitosis in Hep-2 cells. Our previous study determined that $\mathrm{miR}-365 \mathrm{a}-3 \mathrm{p}$ and $\mathrm{miR}-143-5 \mathrm{p}$ were upregulated and miR-494-3p was downregulated in LSCC patients with LNM. In the present study, we successfully transfected Hep-2 cells with miR-365a-3p inhibitor ( $\mathrm{P}<0.001$, Fig. 1A), miR-143-5p inhibitor $(\mathrm{P}<0.001$, Fig. 1B), and miR-494-3p mimics $(\mathrm{P}<0.001$, Fig. 1C) for 48 and $72 \mathrm{~h}$.

We first assessed the roles of these miRNAs in apoptosis in Hep-2 cells by flow cytometric analysis. At 48 and $72 \mathrm{~h}$ after transient transfection, analyses based on Annexin V-FITC/PI apoptosis revealed that with the decreased expression of miR-365a-3p, the number of Hep-2 cells that expressed apoptotic protein Annexin $\mathrm{V}$ was elevated (representative data are shown in Fig. 2A). The result indicates that miR-365a-3p inhibitor significantly facilitated the apoptosis of Hep- 2 cells at 48 and $72 \mathrm{~h}(\mathrm{P}<0.001$, Fig. $2 \mathrm{~B})$. In contrast,
miR-143-5p inhibitor and miR-494-3p mimics had no effect on Hep-2 cells (Fig. 3).

To characterize the influences of these miRNAs on the cell cycle, we used flow cytometry with PI staining to measure the DNA content in order to analyze the cell cycle distribution in Hep-2 cells. The percentages of cells in the S1 phase infected with the NC-inhibitor at 24 and $48 \mathrm{~h}$ after transfection were 36.2 and $37.7 \%$ and with the miR-365a-3p inhibitor were 28.6 and $30.6 \%$, respectively (representative data are shown in Fig. 2C). These findings suggest that the miR-365a-3p inhibitor significantly suppressed cell cycle progression in Hep-2 cells, as shown by the histogram in Fig. $2 \mathrm{D}(\mathrm{P}=0.002)$. However, Hep-2 cells infected with the miR-143-5p inhibitor and the miR-494-3p mimics showed no significant differences compared with the homologous controls (Fig. 4).

miRNA-365 promotes migration and invasion of Hep-2 cells. To determine the biological functions of these miRNA mimics and inhibitors in LSCC metastasis, we performed Transwell migration and invasion assays on Hep-2 cells transfected with miR-365a-3p inhibitor, miR-143-5p inhibitor, and miR-494-3p mimics at $24 \mathrm{~h}$ after transfection. As shown in Fig. 5A, transfection of the miR-365a-3p inhibitor led to 
A

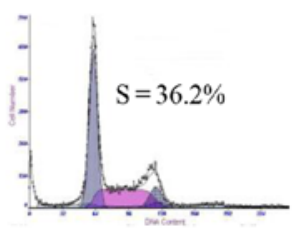

NC-inhibitor $(24 \mathrm{~h})$

B

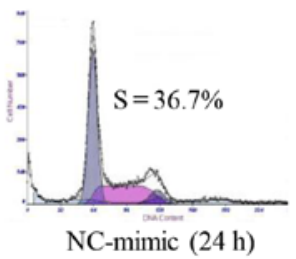

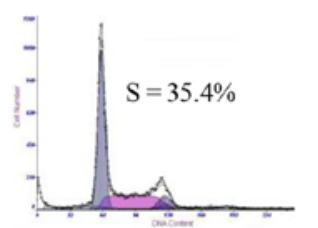

miR-143-5p inhibitor $(24 \mathrm{~h})$

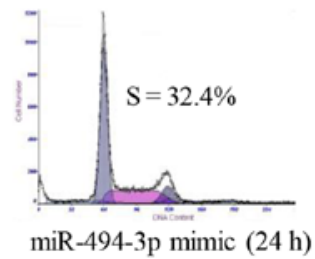

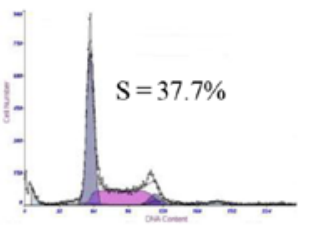

NC-inhibitor $(48 \mathrm{~h})$

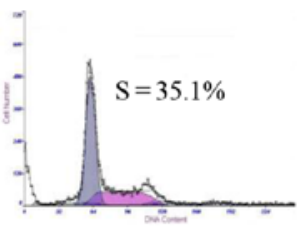

miR-143-5p inhibitor $(48 \mathrm{~h})$
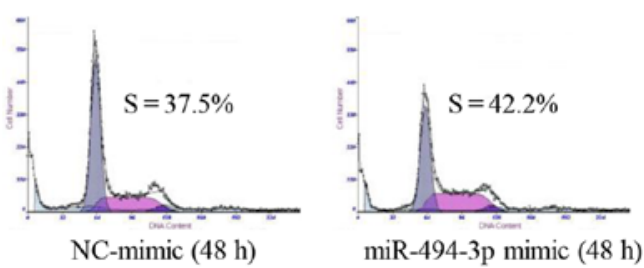

$\mathrm{D}$

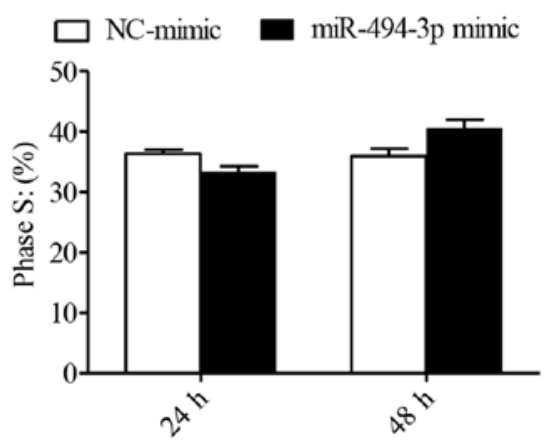

Figure 4. The mitosis assay of infected Hep-2 cells with miR-143-5p inhibitor and miR-494-3p mimic based on flow cytometry analyses. (A and B) The percentage of Hep-2 cells infected with miR-143-5p inhibitor and miR-494-3p mimic in S1 phase at 24 and $48 \mathrm{~h}$ later. (C and D) The numbers of infected homologous Hep-2 cells in S1 phase were counted and are shown in the histograms.

\section{Migration}

A

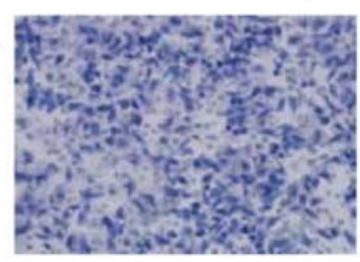

$\mathrm{NC}$-inhibitor

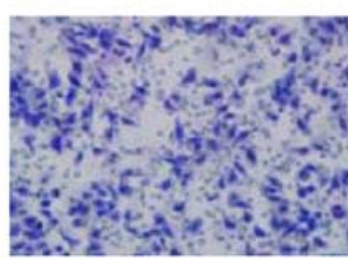

miR-365a-3p inhibitor

\section{Invasion}

B

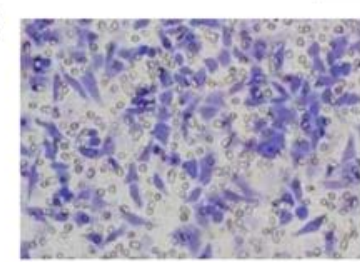

NC-inhibitor

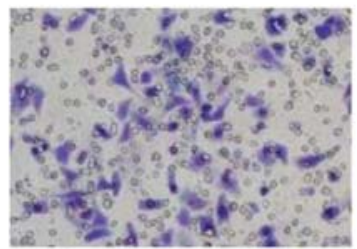

miR-365a-3p inhibitor
$\mathrm{C}$

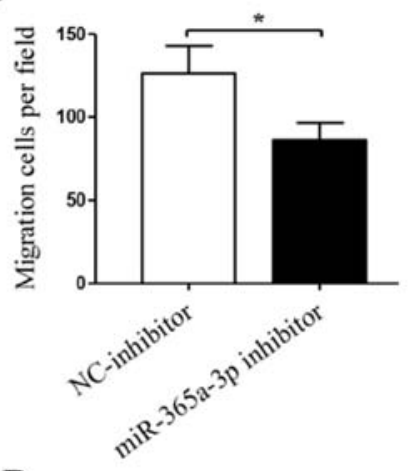

$\mathrm{D}$

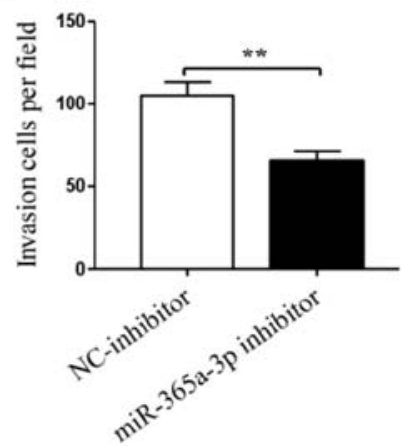

Figure 5. The migration and invasion of Hep-2 cells transfected with miR-365a-3p were measured by the Transwell assay. (A) The migration and (C) invasion of infected Hep-2 cells were measured by the Transwell assay without and with Matrigel, respectively. (B and D) The numbers of Hep-2 cells migrating to and invading the lower chamber were quantified and are shown in histograms $\mathrm{B}$ and $\mathrm{D}$, respectively. ${ }^{*} \mathrm{P}<0.05$ vs. the control; ${ }^{* *} \mathrm{P}<0.01 \mathrm{vs}$. the control. 


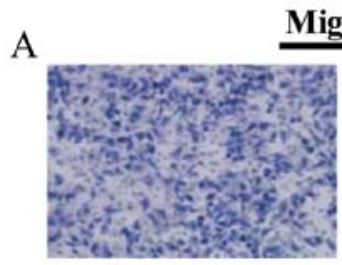

NC-mimic
Migration

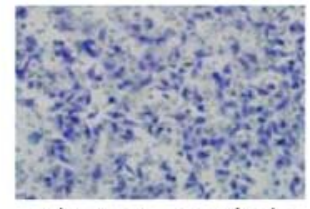

miR-494-3p mimic

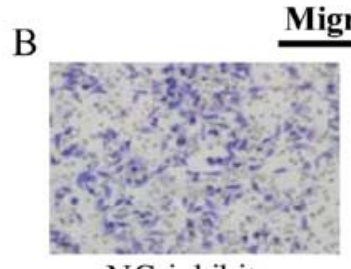

NC-inhibitor

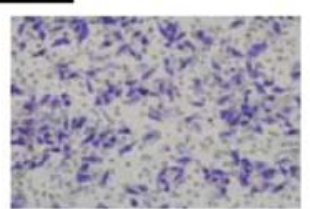

miR-143-5p inhibitor

C

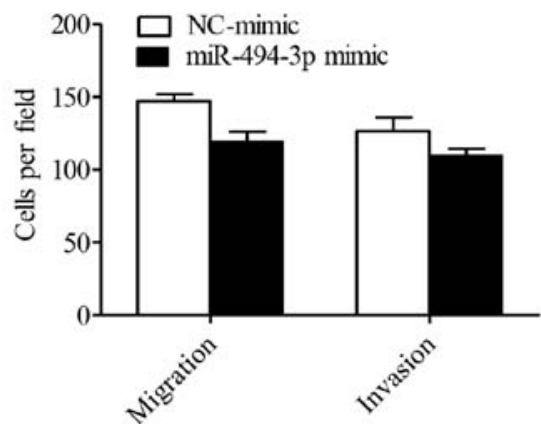

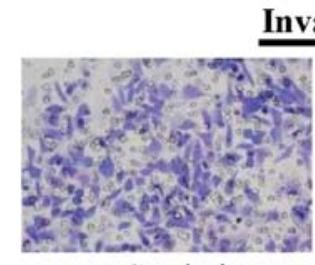

NC-mimic
Invasion

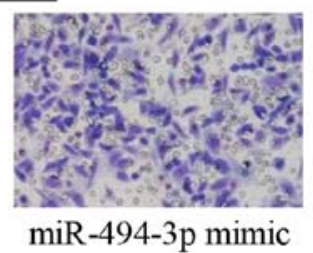

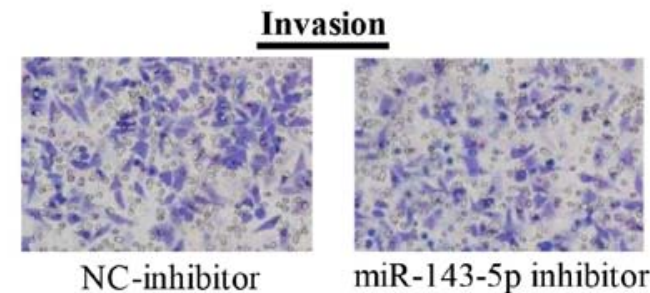

$\mathrm{D}$

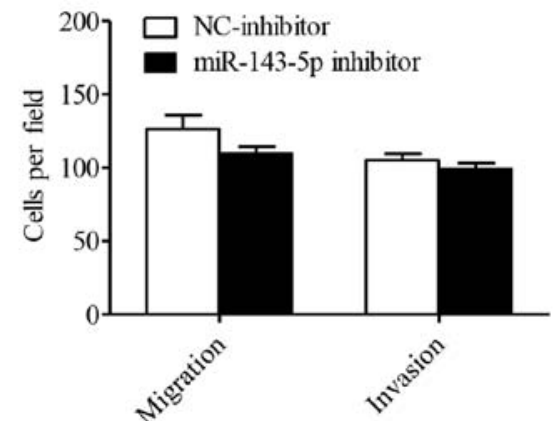

Figure 6. The migration and invasion of Hep-2 cells transfected with miR-143-5p inhibitor and miR-494-3p mimics. (A) The migration and invasion of Hep-2 cells transfected with miR-143-5p inhibitor and (B) miR-494-3p mimics were measured by the Transwell assay without (migration) and with (invasion) Matrigel. (C and D) The numbers of infected Hep-2 cells migrating and invading the lower chamber were quantified and are shown in the histograms.

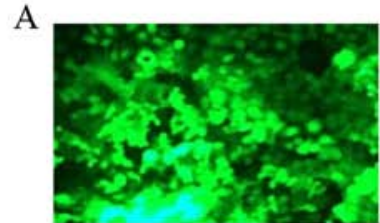

Lv-NC-inhibitor

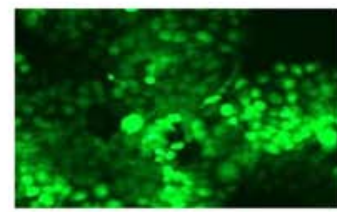

Lv-miR-365a-3p inhibitor

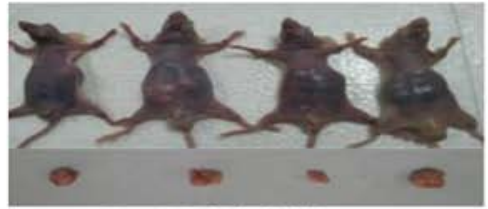

Lv-NC-inhibitor

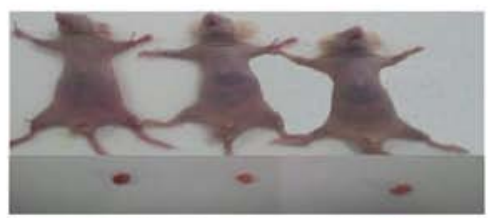

Lv-miR-365a-3p inhibitor
C
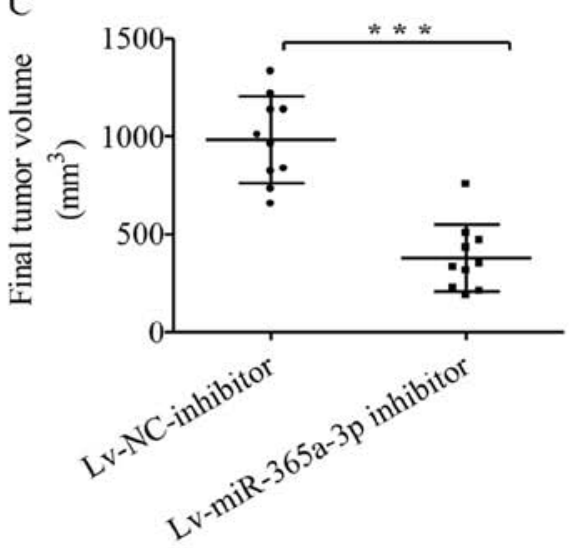

Figure 7. Lv-miR-365a-3p inhibitor suppresses LSCC tumor growth in vivo. (A) Stably transfected Hep-2 cells with Lv-NC-inhibitor and Lv-miR-365a-3p inhibitor. (B) Representative images of nude mice and xenograft tumors after implantation of Hep-2 cells infected with Lv-NC-inhibitor and Lv-miR-365a-3p inhibitor. (C) Scatter plot of the Hep-2 cell tumor volumes following implantation with Lv-NC-inhibitor and Lv-miR-365a-3p inhibitor measured on the day 42 after injection. ${ }^{* * *} \mathrm{P}<0.001$ vs. the control.

significantly decreased cell migration of Hep-2 cells, as shown in the histogram in Fig. 5C ( $\mathrm{P}=0.023)$. Similarly, Transwell invasion assays using Matrigel further demonstrated that only Hep-2 cells transfected with the miR-365a-3p inhibitor were significantly reduced compared to the parental control cells $(\mathrm{P}=0.0024)$ (Fig. 5B and D). As shown in Fig. 6, the
miR-143-5p inhibitor and the miR-494-3p mimics had no significant influence on the cell migration and invasion of Hep-2 cells.

miR-365a-3p promotes LSCC tumor growth and metastasis in vivo. To further view the potential effects of miR-365a-3p, 
A

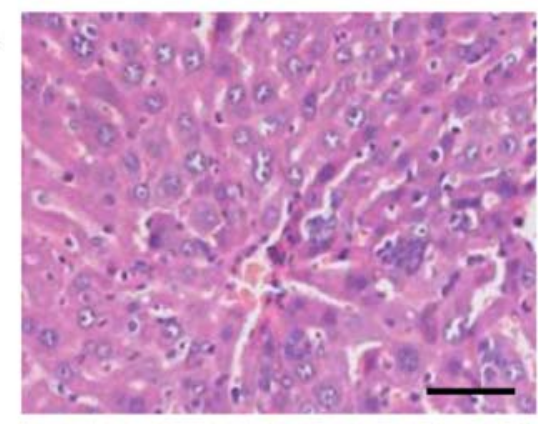

Liver metastasis

C

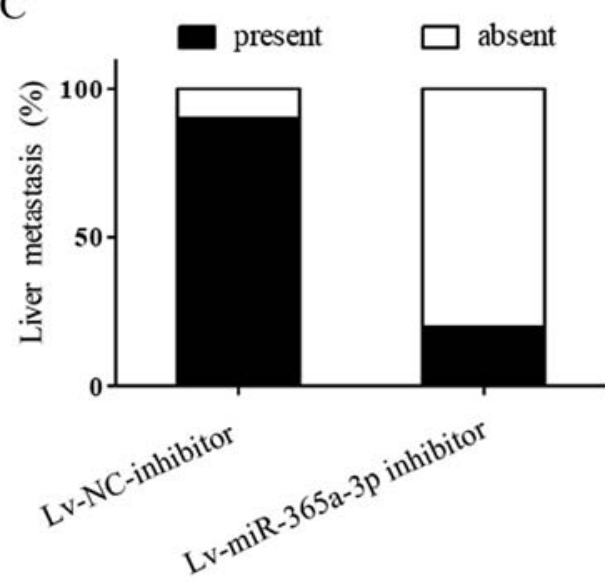

$\mathrm{B}$

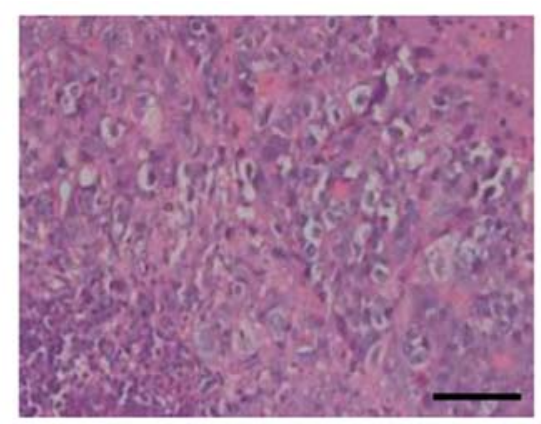

HLN metastasis

$\mathrm{D}$
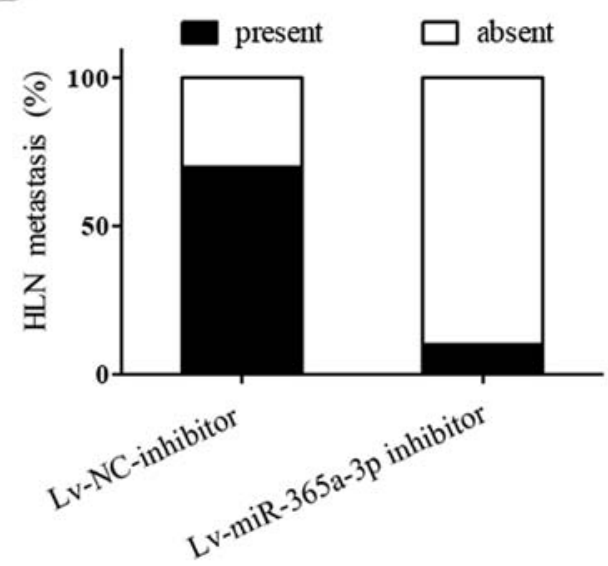

Figure 8. Lv-miR-365a-3p inhibitor suppresses LSCC metastasis in vivo. (A) Liver metastasis and (B) hepatic lymph node (HLN) metastasis in LSCC cells. Hematoxylin and eosin staining with original magnification x200; scale bar, $50 \mu \mathrm{m}$. (C) The percentages of mice with or without liver metastasis were calculated and are shown in the stacked bars. (D) The percentages of mice with or without HLN metastasis were calculated and are shown in the stacked bars.

we established stably transfected Hep-2 cells containing an Lv-NC-inhibitor and an Lv-miR-365a-3p inhibitor (Fig. 7A) and injected the inhibitors into the enterocoelia of nude mice. The mice were euthanized, and the tumors were harvested on day 42 after injection. Tumors formed from Hep-2 cells stably transfected with the Lv-NC-inhibitor grew much faster than tumors from the cells that stably expressed the Lv-miR-365a-3p inhibitor (Fig. 7B). Accordingly, the final tumor volumes in the two groups were calculated and compared, as shown in Fig. 7C $(\mathrm{P}<0.0001$ for the difference).

We further explored the metastasis in vivo using a body vision microscope. We found that tumors were metastasized in the livers (Fig. 8A) and hepatic lymph nodes (Fig. 8B). In particular, metastases to the livers in the Lv-NC-inhibitor group occurred at a significantly higher frequency than those in the Lv-miR-365a-3p inhibitor group. As shown in the stacked bars of Fig. 8C, 9 of 10 mice in the Lv-NC-inhibitor group showed liver metastases, but in the Lv-miR-365a-3p inhibitor group only 2 of 10 mice showed liver metastases. As expected, there was a similar trend of metastases in the hepatic lymph nodes in the Lv-NC-inhibitor and the Lv-miR-365a-3p inhibitor groups: 7 in 10 mice and only 1 in 10 mice showed hepatic lymph node metastases, respectively (Fig. 8D). These findings indicate that miR-365a-3p promotes LSCC tumor growth and metastases in vivo.

miR-365a-3p inhibitor downregulates p-AKT (Ser473). ERK and AKT play key roles in proliferation, migration, and inva- sion in malignant tumor cells. Accordingly, we performed western blotting to examine the protein levels of p-ERK1/2, p-AKT (Thr308), and p-AKT (Ser473). The results showed that the levels of p-ERK1/2 have no significant difference after miR-365a-3p inhibitor transfection compared with the levels of NC-inhibitor ( $\mathrm{P}=0.082$, Fig. 9A). Interestingly, miR-365a-3p inhibitor significantly decreased the expression of $\mathrm{p}-\mathrm{AKT}$ (Ser473) but not p-AKT (Thr308) compared to the expression in the Hep-2 cells transfected with matched NC-inhibitor miRNA (Ser473, P<0.001; Thr308, P<0.245, Fig. 9B).

\section{Discussion}

Aberrant expression of miRNAs has been demonstrated to contribute to LSCC tumorigenesis and progression $(13,15)$. For the first time, this study analyzed the roles of miR-365a-3p, miR-143-5p, and miR-494-3p in Hep-2 cells. Because of the overexpression of miR-365a-3p and miR-143-5p and the lower expression of miR-494-3p in LSCC patients with LNM (14), we transfected Hep-2 cells with miR-365a-3p inhibitor, miR143-5p inhibitor, and miR-494-3p mimics. Interestingly, the loss-of-function results demonstrated that only the miR-365a-3p inhibitor significantly facilitated apoptosis and suppressed mitosis, migration, and invasion of Hep-2 cells, and we found that miR-365a-3p promotes LSCC xenograft tumor growth and metastasis in vivo. These data suggest that miR-365a-3p may be an oncomiR in LCSS, and miR-365a-3p inhibition may have potential in the treatment of laryngeal carcinoma. 
A

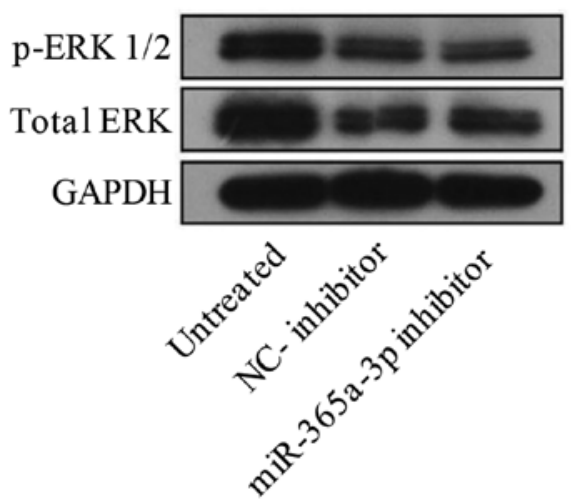

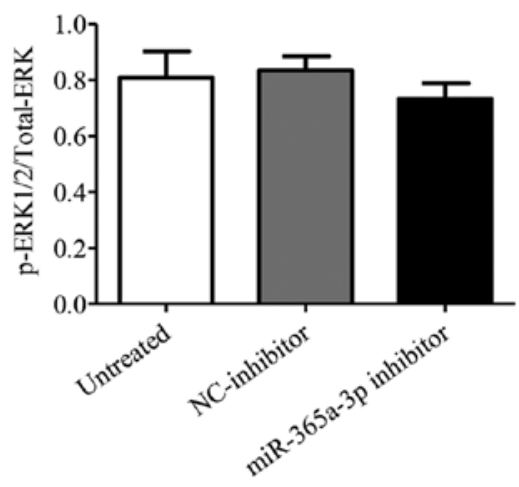

B

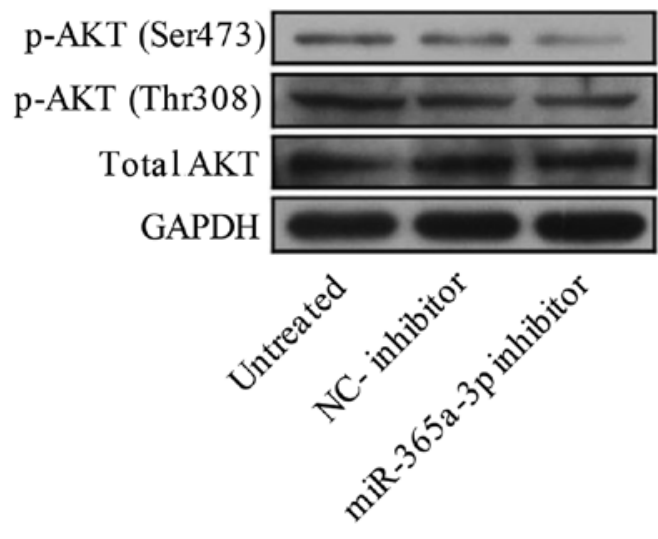

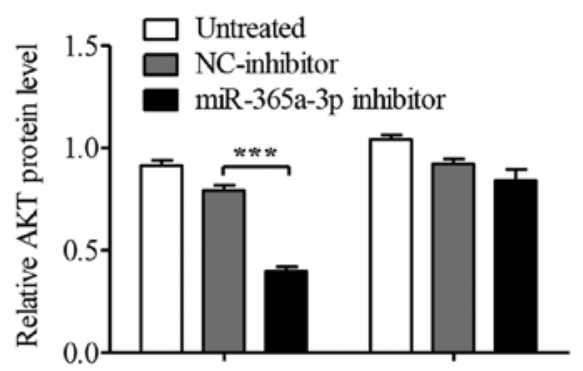

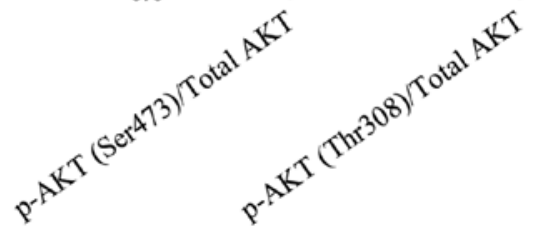

Figure 9. miR-365a-3p inhibitor downregulates the expression of p-AKT (Ser473) as determined using western blot analyses. (A) miR-365a-3p inhibitor did not affect p-ERK1/2 or total ERK expression. (B) miR-365a-3p inhibitor downregulated p-AKT (Ser473) protein levels but did not affect p-AKT (Thr308) or total AKT expression. GAPDH was the endogenous normalizer. ${ }^{* * *} \mathrm{P}<0.001$ vs. the control.

The mature miR-365 is derived from two separate RNA precursors, hsa-miR-365a (previously named hsa-miR-365-1, MI0000767) and hsa-miR-365b (previously named hsa-miR-365-2, MI0000769), and mature hsa-miR-365a-3p (MIMAT0000710) is sheared from hsa-miR-365a (www. mirbase.org). miR-365 has been confirmed to act as an oncogene or tumor suppressor in different carcinomas $(16,17)$, although this finding has not previously been reported in LSCC. Gastaldi et al (18) found that the expression of miR-365a was decreased in chemically-induced mouse skin carcinomas, which suggests a tumor suppressor role of miR-365a although miR-365a-3p has been found to be an oncogene (19). A recent study revealed that miR-365b was upregulated in cutaneous squamous cell carcinoma and induced subcutaneous tumors in vivo (16). The study also found that anti-miR-365b oligonucleotide inhibited cutaneous tumor formation in nude mice, along with apoptosis and G1 phase arrest in cancer cells. Hamada et al also found that miR-365 was highly expressed in ductal adenocarcinoma of the pancreas and contributed to the epithelial-mesenchymal transition. miR-365 can target apoptosis-promoting protein BAX and adaptor protein Src homology 2 domain containing 1, and then influences the survival of pancreatic cancer cells (20). Obviously, the expression levels of miR-365 are diverse in different malignant tumors. For example, in human gastric cancer the low expression of miR-365 correlated with poorly differentiated histology, advanced stage, and deep invasion, as well as the deregulation of phosphorylated Akt, p53, and cyclin D1. In mouse gastric cancers, the activation of Akt led to downregulated transcription of miR-365 and promoted gastric cancer cell proliferation (17). These data demonstrate that miR-365 was downregulated in gastric cancer. In addition, miR-365 was also found to be downregulated and accompanied the overexpression of NKX2-1 protein in lung cancer tissues. Furthermore, the miR-365 mimic significantly reduced the proliferation of lung cancer cells (21). The discrepancy of these findings may be due to the dynamic tumor microenvironment, stages of progression, and different types of cancers studied.

PI3K/AKT and MAPK signaling pathways are often hyperactivated in many malignant tumors, including LSCC, and the dependence of carcinoma cells on the two activated pathways has been used successfully in the clinic (22-25). AKT and ERK play key roles in the PI3K/AKT pathway and MAPK pathway, respectively $(26,27)$. Recent studies have also found that AKT and ERK are highly correlated with miRNAs in various human malignancies (28-30). Therefore, to explore the underlying signaling pathways of miR-365a-3pinduced LCSS cell proliferation, migration, and invasion, we examined the expression of p-AKT (Thr308), p-AKT (Ser473), and $\mathrm{p}-\mathrm{ERK} 1 / 2$. The data showed that only the expression of p-AKT (Ser473) was significantly influenced by the miR365a-3p inhibitor. The serine/threonine kinase Akt encoded by the protein kinase B gene is a downstream effector of 
PI3K. Studies have shown that activation of Akt signaling is responsible for cancer cell proliferation, invasiveness, and metastasis (17,31). AKT activation is initiated by docking of the PH domain of AKT to PIP3 on the cellular membrane, exposing two critical amino acid residues for phosphorylation (32). Both phosphorylation events, of Ser473 by the protein kinase PDK1 and of Thr308 by the mTORC 2 complex, are required for full activation of AKT (33). However, the levels of AKT phosphorylation on either Ser473 or Thr308 correlate differently with tumor cell growth and proliferation in distinct carcinomas (34-37). In our study, p-AKT (Ser473) was significantly decreased by the miR-365a-3p inhibitor, but p-AKT (Ser308) had no such influence. The data suggested that miR-365a-3p can mediate the PI3K/AKT signaling pathway by upregulating p-AKT (Ser473) to promote growth and metastasis in LSCC. This result is consistent with a recent study that showed that patients with head and neck squamous cell carcinomas with higher levels of p-AKT (Ser473) had worse survival, but the survival of patients with higher levels of p-AKT (Thr308) was not affected (25).

Dysregulated miR-143 and miR-494 have important roles in many carcinomas $(38,39)$, although this association has not been reported in LSCC patients. A recent study of epithelial cancers, including esophagus and lung cancer, indicated that downregulation of miR-143 contributes to epithelial cancer development, and its re-expression suppresses cellular proliferation and triggers apoptosis of epithelial cancer cells (38). miR-494 was reported to be significantly upregulated and to negatively modulate the expression of its target gene PTEN, in human cervical cancer cell lines and tumor samples (39). These findings identified the essential roles that miR-143 and miR-494 play in many carcinomas. However, our experiment shows that the two miRNAs did not have significant influence on Hep-2 cell apoptosis, mitosis, migration, and invasion in vitro. This is partly explained by the observation that miRNAs may have different roles cancer type-dependently.

In view of the low 5-year survival rate and the limited improvements in the treatment of LSCC during the past 20 years $(1,2)$, finding novel molecular therapeutic targets for treating LSCC is essential. Because miRNAs play pivotal roles in the development of carcinoma, it is conceivable that miRNA mimics or inhibitors may become a new class of molecular target-based therapies for various cancers (40-42). Their effects and regulatory mechanisms in LSCC remain uncertain, but the present study revealed that miR-365a-3p, a novel oncomiR, activates the PI3K/AKT signaling pathway via p-AKT (Ser473) and can promote LSCC growth and metastasis. Therefore, miR-365a-3p may become a potential therapeutic target for the treatment of LSCC.

\section{Acknowledgements}

This study was supported by the National Natural Science Foundation of China (\#81071785), the Clinical Research Special Foundation by the Wu Jieping Medical Foundation (320.6750.12398), the Beijing Municipal Science and Technology Project (D131100005313014), the Scientific Research Project of Beijing Children's Hospital, Capital Medical University (2012ZD01), and the Science and Technology Planning Project of Beijing (Z151100003715006).

\section{References}

1. Siegel RL, Miller KD and Jemal A: Cancer statistics, 2015. CA Cancer J Clin 65: 5-29, 2015.

2. Megwalu UC and Sikora AG: Survival outcomes in advanced laryngeal cancer. JAMA Otolaryngol Head Neck Surg 140: 855-860, 2014.

3. Connor KL, Pattle S, Kerr GR and Junor E: Treatment, comorbidity and survival in stage III laryngeal cancer. Head Neck 37: 698-706, 2015.

4. Berindan-Neagoe I, Monroig PC, Pasculli B and Calin GA: MicroRNAome genome: A treasure for cancer diagnosis and therapy. CA Cancer J Clin 64: 311-336, 2014.

5. Valeri N, Braconi C, Gasparini P, Murgia C, Lampis A, Paulus-Hock V, Hart JR, Ueno L, Grivennikov SI, Lovat F, et al: MicroRNA-135b promotes cancer progression by acting as a downstream effector of oncogenic pathways in colon cancer. Cancer Cell 25: 469-483, 2014.

6. Drayton RM, Dudziec E, Peter S, Bertz S, Hartmann A, Bryant HE and Catto JW: Reduced expression of miRNA-27a modulates cisplatin resistance in bladder cancer by targeting the cystine/glutamate exchanger SLC7A11. Clin Cancer Res 20: 1990-2000, 2014.

7. Kong YW, Ferland-McCollough D, Jackson TJ and Bushell M: microRNAs in cancer management. Lancet Oncol 13: e249-e258, 2012.

8. Ouyang H, Gore J, Deitz S and Korc M: microRNA-10b enhances pancreatic cancer cell invasion by suppressing TIP30 expression and promoting EGF and TGF- $\beta$ actions. Oncogene 33: 4664-4674, 2014.

9. Zhou J, Zhang M, Huang Y, Feng L, Chen H, Hu Y, Chen H, Zhang K, Zheng L and Zheng S: MicroRNA-320b promotes colorectal cancer proliferation and invasion by competing with its homologous microRNA-320a. Cancer Lett 356: 669-675, 2015.

10. Tu Y, Gao X, Li G, Fu H, Cui D, Liu H, Jin W and Zhang Y: MicroRNA-218 inhibits glioma invasion, migration, proliferation, and cancer stem-like cell self-renewal by targeting the polycomb group gene Bmi1. Cancer Res 73: 6046-6055, 2013.

11. Yongchun Z, Linwei T, Xicai W, Lianhua Y, Guangqiang Z, Ming Y, Guanjian L, Yujie L and Yunchao H: MicroRNA-195 inhibits non-small cell lung cancer cell proliferation, migration and invasion by targeting MYB. Cancer Lett 347: 65-74, 2014.

12. Sun X, Liu B, Zhao XD, Wang LY and Ji WY: MicroRNA-221 accelerates the proliferation of laryngeal cancer cell line Hep-2 by suppressing Apaf-1. Oncol Rep 33: 1221-1226, 2015.

13. Tian Y, Fu S, Qiu GB, Xu ZM, Liu N, Zhang XW, Chen S, Wang Y, Sun KL and Fu WN: MicroRNA-27a promotes proliferation and suppresses apoptosis by targeting PLK2 in laryngeal carcinoma. BMC Cancer 14: 678-689, 2014.

14. Tai J, Xiao X, Huang ZG, Yu ZK, Chen XH, Zhou WG, Chen XJ, Rao YS, Fang JG and Ni X: MicroRNAs regulate epithelial-mesenchymal transition of supraglottic laryngeal cancer. Zhonghua Er Bi Yan Hou Tou Jing Wai Ke Za Zhi 48: 499-503, 2013 (In Chinese).

15. Xu Y, Wang K, Gao W, Zhang C, Huang F, Wen S and Wang B: MicroRNA-106b regulates the tumor suppressor RUNX3 in laryngeal carcinoma cells. FEBS Lett 587: 3166-3174, 2013.

16. Zhou M, Liu W, Ma S, Cao H, Peng X, Guo L, Zhou X, Zheng L, Guo L, Wan M, et al: A novel onco-miR-365 induces cutaneous squamous cell carcinoma. Carcinogenesis 34: 1653-1659, 2013.

17. Guo SL, Ye H, Teng Y, Wang YL, Yang G, Li XB,Zhang C, Yang X, Yang ZZ and Yang X: Akt-p53-miR-365-cyclin D1/cdc25A axis contributes to gastric tumorigenesis induced by PTEN deficiency. Nat Commun 4: 2544-2554, 2013.

18. Gastaldi C, Bertero T, Xu N, Bourget-Ponzio I, Lebrigand K, Fourre S, Popa A, Cardot-Leccia N, Meneguzzi G, Sonkoly E, et al: miR-193b/365a cluster controls progression of epidermal squamous cell carcinoma. Carcinogenesis 35: 1110-1120, 2014.

19. Tarasov VA, Matishov DG, Shin EF, Boǐko NV, Timoshkina NN, Makhotkin MA, Lomonosov AM and Kirpiĭ AA: Inheritable changes in miRNAs expression in HeLa cells after X-ray and mitomycin C treatment. Genetika 50: 909-917, 2014 (In Russian).

20. Hamada S, Masamune A, Miura S, Satoh K and Shimosegawa T: miR-365 induces gemcitabine resistance in pancreatic cancer cells by targeting the adaptor protein SHC1 and pro-apoptotic regulator BAX. Cell Signal 26: 179-185, 2014.

21. Kang SM, Lee HJ and Cho JY: MicroRNA-365 regulates NKX2-1, a key mediator of lung cancer. Cancer Lett 335: 487-494, 2013. 
22. Smith MP, Sanchez-Laorden B, O'Brien K, Brunton H, Ferguson J, Young H, Dhomen N, Flaherty KT, Frederick DT, Cooper ZA, et al: The immune microenvironment confers resistance to MAPK pathway inhibitors through macrophage-derived TNF $\alpha$. Cancer Discov 4: 1214-1229, 2014.

23. Mittal S, Sharma A, Balaji SA, Gowda MC, Dighe RR, Kumar RV and Rangarajan A: Coordinate hyperactivation of Notch1 and Ras/MAPK pathways correlates with poor patient survival: Novel therapeutic strategy for aggressive breast cancers. Mol Cancer Ther 13: 3198-3209, 2014.

24. Bjerke GA, Yang CS, Frierson HF, Paschal BM and Wotton D: Activation of Akt signaling in prostate induces a TGF $\beta$-mediated restraint on cancer progression and metastasis. Oncogene 33 3660-3667, 2014

25. Freudlsperger C, Horn D, Weißfuß S, Weichert W, Weber KJ, Saure D, Sharma S, Dyckhoff G, Grabe N, Plinkert P, et al: Phosphorylation of AKT(Ser473) serves as an independent prognostic marker for radiosensitivity in advanced head and neck squamous cell carcinoma. Int J Cancer 136: 2775-2785, 2015.

26. Fresno Vara JA, Casado E, de Castro J, Cejas P, Belda-Iniesta C and González-Barón M: PI3K/Akt signalling pathway and cancer. Cancer Treat Rev 30: 193-204, 2004.

27. McKay MM and Morrison DK: Integrating signals from RTKs to ERK/MAPK. Oncogene 26: 3113-3121, 2007.

28. Du J, Liu S, He J, Liu X, Qu Y, Yan W, Fan J, Li R, Xi H, Fu W, et al: MicroRNA-451 regulates stemness of side population cells via PI3K/Akt/mTOR signaling pathway in multiple myeloma. Oncotarget 6: 14993-15007, 2015.

29. Ihle MA, Trautmann M, Kuenstlinger H, Huss S, Heydt C, Fassunke J, Wardelmann E, Bauer S, Schildhaus HU, Buettner R, et al: miRNA-221 and miRNA-222 induce apoptosis via the KIT/AKT signalling pathway in gastrointestinal stromal tumours. Mol Oncol 9: 1421-1433, 2015.

30. Wang W, Ren F, Wu Q, Jiang D, Li H and Shi H: MicroRNA-497 suppresses angiogenesis by targeting vascular endothelial growth factor A through the PI3K/AKT and MAPK/ERK pathways in ovarian cancer. Oncol Rep 32: 2127-2133, 2014.

31. Xue G, Restuccia DF, Lan Q, Hynx D, Dirnhofer S, Hess D, Rüegg $\mathrm{C}$ and Hemmings BA: Akt/PKB-mediated phosphorylation of Twist 1 promotes tumor metastasis via mediating cross-talk between PI3K/Akt and TGF- $\beta$ signaling axes. Cancer Discov 2: 248-259, 2012.

32. Stephens L, Anderson K, Stokoe D, Erdjument-Bromage H, Painter GF, Holmes AB, Gaffney PR, Reese CB, McCormick F, Tempst P, et al: Protein kinase B kinases that mediate phosphatidylinositol 3,4,5-trisphosphate-dependent activation of protein kinase B. Science 279: 710-714, 1998.
33. Freudlsperger C, Burnett JR, Friedman JA, Kannabiran VR, Chen $\mathrm{Z}$ and Van Waes C: EGFR-PI3K-AKT-mTOR signaling in head and neck squamous cell carcinomas: Attractive targets for molecular-oriented therapy. Expert Opin Ther Targets 15: 63-74, 2011.

34. Tsurutani J, Fukuoka J, Tsurutani H, Shih JH, Hewitt SM, Travis WD, Jen J and Dennis PA: Evaluation of two phosphorylation sites improves the prognostic significance of Akt activation in non-small-cell lung cancer tumors. J Clin Oncol 24: 306-314, 2006.

35. Lin A, Piao HL, Zhuang L, Sarbassov D, Ma L and Gan B: FoxO transcription factors promote AKT Ser473 phosphorylation and renal tumor growth in response to pharmacologic inhibition of the PI3K-AKT pathway. Cancer Res 74: 1682-1693, 2014.

36. Beck JT, Ismail A and Tolomeo C: Targeting the phosphatidylinositol 3-kinase (PI3K)/AKT/mammalian target of rapamycin (mTOR) pathway: An emerging treatment strategy for squamous cell lung carcinoma. Cancer Treat Rev 40: 980-989, 2014.

37. Gallay N, Dos Santos C, Cuzin L, Bousquet M, Simmonet Gouy V, Chaussade C, Attal M, Payrastre B, Demur C and Récher C: The level of AKT phosphorylation on threonine 308 but not on serine 473 is associated with high-risk cytogenetics and predicts poor overall survival in acute myeloid leukaemia. Leukemia 23: 1029-1038, 2009.

38. Zhang J, Sun Q, Zhang Z, Ge S, Han ZG and Chen WT: Loss of microRNA-143/145 disturbs cellular growth and apoptosis of human epithelial cancers by impairing the MDM2-p53 feedback loop. Oncogene 32: 61-69, 2013.

39. Yang YK, Xi WY, Xi RX, Li JY, Li Q and Gao YE: MicroRNA-494 promotes cervical cancer proliferation through the regulation of PTEN. Oncol Rep 33: 2393-2401, 2015.

40. Zhao L, Liu W, Xiao J and Cao B: The role of exosomes and 'exosomal shuttle microRNA' in tumorigenesis and drug resistance. Cancer Lett 356: 339-346, 2015.

41. Won YS, Jeong JS, Kim SJ, Ju MH and Lee SW: Targeted anticancer effect through microRNA-181a regulated tumor-specific hTERT replacement. Cancer Lett 356: 918-928, 2015.

42. Serpico D, Molino L and Di Cosimo S: microRNAs in breast cancer development and treatment. Cancer Treat Rev 40 595-604, 2014 